\title{
The JRC Multidisciplinary Research Data Infrastructure
}

\author{
Anders Friis-Christensen \\ European Commission, Joint Research Centre (JRC), \\ Directorate Growth \& Innovation, Digital Economy Unit \\ anders.friis@ec.europa.eu \\ Chrisa Tsinaraki \\ European Commission, Joint Research Centre (JRC), \\ Directorate Growth \& Innovation, Digital Economy Unit \\ chrysi.tsinaraki@ec.europa.eu
}

\begin{abstract}
This paper presents the approach adopted by the European Commission's Joint Research Centre (JRC) in order to facilitate open access to its research data crated as support for EU policies, which is also in line with the general Open Data trend. The paper presents various initiatives and incentives that are put in place at the JRC in order to progressively implement a multi-disciplinary research data infrastructure for the fulfilment of the corporate data policy goals. These include, among others, the JRC metadata schema that is developed with the aim to harmonise the way data are described, as well as the architecture of a data infrastructure designed to support the multidisciplinary nature of the JRC activities.
\end{abstract}

\section{CCS CONCEPTS}

- Information systems $\rightarrow$ Data management systems; Enterprise information systems; Enterprise applications;

\section{KEYWORDS}

research data, data infrastructure, data policy, open data, semantic Web, linked data, interoperability, reproducibility

\section{ACM Reference format:}

Anders Friis-Christensen, Andrea Perego, Chrisa Tsinaraki, and Lorenzino Vaccari. 2017. The JRC Multidisciplinary Research Data Infrastructure. In Proceedings of The 19th International Conference on Information Integration and Web-based Applications \& Services, Salzburg, Austria, December 4-6, 2017 (iiWAS '17), 5 pages.

https://doi.org/10.1145/3151759.3151810

\section{INTRODUCTION}

The European Commission (EC) seeks to provide a transparent and coherent policymaking that is based on sound and robust scientific evidence [8]. Several regulatory frameworks and policies are in ${ }^{*}$ The views expressed are purely those of the author and may not in any circumstances
be regarded as stating an official position of the European Commission.

Permission to make digital or hard copies of part or all of this work for personal or classroom use is granted without fee provided that copies are not made or distributed for profit or commercial advantage and that copies bear this notice and the full citation on the first page. Copyrights for third-party components of this work must be honored.

For all other uses, contact the owner/author(s).

iiWAS '17, December 4-6, 2017, Salzburg, Austria

(C) 2017 Copyright held by the owner/author(s)

ACM ISBN 978-1-4503-5299-4/17/12.

https://doi.org/10.1145/3151759.3151810

\author{
Andrea Perego \\ European Commission, Joint Research Centre (JRC), \\ Directorate Growth \& Innovation, Digital Economy Unit \\ andrea.perego@ec.europa.eu

\section{Lorenzino Vaccari} \\ European Commission, Joint Research Centre (JRC), \\ Directorate Growth \& Innovation, Digital Economy Unit \\ lorenzino.vaccari@ec.europa.eu
}

place to ensure that this goal is achieved. An important policy commitment for the European Commission (EC) and the Joint Research Centre (JRC) in this regard includes the Commission Decision on the reuse of Commission documents (2011/833/EU) [4]. As with other research funded in the context of the EU Programme Horizon 2020 (https://ec.europa.eu/programmes/horizon2020/) [10], the JRC has the additional commitment to ensure access to both scientific publications and to the data produced as part of its research. Further commitments include: Commission communication on better access to scientific information (COM (2012) 401) [6], Commission recommendation on access to and preservation of scientific information (COM (2012) 4890) [5] and the EU implementation of the G8 Open Data Charter [7].

The overall mission of the JRC is to support EU policies with independent evidence throughout the whole policy life-cycle. The activities of the JRC span many different research areas, that address and contribute to a healthy and safe environment, secure energy supplies, sustainable mobility and consumer health and safety. Thus, the JRC is a multidisciplinary research organisation and the diversity of its research activities poses challenges in the management of data, since different scientific disciplines have their own traditions, standards and best-practices on how to manage and disseminate research information.

In order to provide a basis for better management of data, in 2014 the JRC developed a corporate data policy [2], driven by the need of transparency and to facilitate open access to research data, in line with the general Open Data trend.

In this paper, we present the various initiatives and incentives that are put in place at the JRC in order to progressively implement a multi-disciplinary research data infrastructure for the fulfilment of the JRC Data Policy goals. More precisely, after providing an overview of the JRC Data Policy (Section 2), we illustrate the requirements and the design principles of the JRC metadata schema (Section 3) and describe the architecture of the JRC Data Infrastructure (Section 4). Finally, we summarise the work reported in the paper (Section 5) and outline future work.

\section{BACKGROUND}

Driven by the aims of transparency, efficiency and contribution to innovation, and in order to provide a basis for better management of data, the JRC published a Data Policy [2] in 2015, which is built on the following principles: 
- Open Data principles: Free, full, open and timely access to JRC data as default rule.

- Data acquisition principles: Negotiating restriction-free access and use of licensed data.

- Data management principles: Metadata, storage, accessibility.

- Implementation principles: Identification of responsible entities, guidelines, data management plans, monitoring of implementation.

The Open Data principles of the JRC Data Policy are in line with the Commission Decision on the reuse of Commission documents (2011/833/EU) [4]. Since much data at the JRC is created based on acquired third party data, the JRC Data Policy also addresses data acquisition principles. This is done mainly with the aim of avoiding restrictions for the JRC/Commission on the reuse of third party data, where feasible.

In order to operationalise its implementation, the JRC Data Policy requires that principles on data management are further elaborated in associated Data Policy Implementation Guidelines. Proper corporate data management is inherent to the successful implementation of the JRC Data Policy, and needs to address all aspects of the data life-cycle management. The JRC Data Policy Implementation Guidelines address additional issues such as governance and common infrastructure components supporting data life-cycle management.

Apart from the legal obligations of the JRC on sharing its research information, one of the overall aims of the JRC Data Policy is to further ensure transparency of the data policy development lifecycle to which the JRC is providing evidence. This includes that data used in models in order to derive scientific evidence, such as impact assessments, need to be well documented and, more importantly, accessible. This shall ensure the ability to reproduce or at least replicate the evidence.

One of the main challenges of the implementation of the JRC Data Policy is to ensure the commitment of the JRC scientists. Undoubtedly, the pledge towards open access of research data requires additional resources and changes to existing workflows and procedures. Changes that have impact on existing cultures highlight the need for commitments at all levels. Thus, it is important for the successful implementation of the JRC Data Policy, to create incentives and benefits for the individual scientists.

A tangible and already available outcome of the implementation of the JRC Data Policy, is the single access point that provides access to the JRC data, namely the JRC Data Catalogue that is available at: http://data.jrc.ec.europa.eu.

\section{METADATA FOR A MULTI-DISCIPLINARY DATA INFRASTRUCTURE}

Metadata play a fundamental role in the JRC Data Infrastructure, and are meant to address the following main requirements:

- Ensuring data documentation and inventory, related to the purposes of the JRC Data Catalogue-as per the JRC Data Policy.

- Enabling data discovery for data consumers, both (a) for the general public and (b) internally to the JRC, the Commission and the other EU institutions and bodies.

- Publication on other data portals operated by EU institutions and bodies, and in particular on the EU Open Data Portal
(ODP) - as per the requirement of the Commission Decision on the reuse of Commission documents (2011/833/EU) [4].

- Supporting data citation by using current best practices and making data recognised as a scientific output. Support for data citation is an important aspect, since it creates an incentive for the JRC scientists to be committed to the implementation of JRC Data Policy; thus, it helps in dealing with one of the main challenges that we are facing.

- Building the data context, by linking datasets to related resources. These include dataset authors, scientific publications concerning the relevant dataset, input data, the model(s) used for creating the dataset, as well as legislation and impact assessments.

The multidisciplinary nature of JRC datasets makes it difficult to define a common metadata schema and its sustainability in the long-term-in particular, with respect to backward compatibility.

Based on the requirements outlined above, and following common practices, the design of the JRC metadata schema is following a modular approach consisting of:

- A core profile, defining the elements that should be common to all metadata records.

- A set of extensions, defining elements specific to given domains (geospatial, statistical, etc.).

As far as the core profile is concerned, the reference standards taken into account are DCAT-AP [11] (the de facto EU standard metadata interchange format, based on the W3C Data Catalog vocabulary [15]) and DataCite [1] (the de facto international metadata standard for data citation).

Domain specific extensions will be based on existing metadata standards, such as ISO 19115 / INSPIRE [3, 14] for geospatial metadata, and SDMX [13] for statistical metadata. Whenever possible, such profiles will be contributed and/or aligned with existing standardisation efforts at the EU and international level, to ensure interoperability of JRC metadata with other infrastructures. As an example, the JRC geospatial metadata profile is based on GeoDCATAP $[12,16]$, which extends DCAT-AP with metadata elements from ISO 19115 and INSPIRE.

The JRC metadata schema is an extension to DCAT-AP (DCAT application profile for data portals in Europe) [11], defined to ensure compliance with the DataCite metadata schema [1]. In order to identify how DCAT-AP needed to be extended to enable data citation, we carried out a preliminary study, resulting in the definition of mappings from DataCite to DCAT-AP [17, 18], accompanied with their XSLT-based implementation.

Based on this work, the JRC metadata schema has been extended with two entities not included in DCAT-AP:

Contributor It is meant to denote the authors of a dataset.

Publication A document (such as a scientific publication or technical report) related to and/or citing the dataset. This is a specialisation of the DCAT-AP notion of "related resource"which is how dataset-related publications can be modelled in DCAT-AP.

The diagram in Figure 1 depicts the main resources described in the JRC metadata schema, along with their attributes and relationships. In the figure, "other resources" is a catch-all category that 


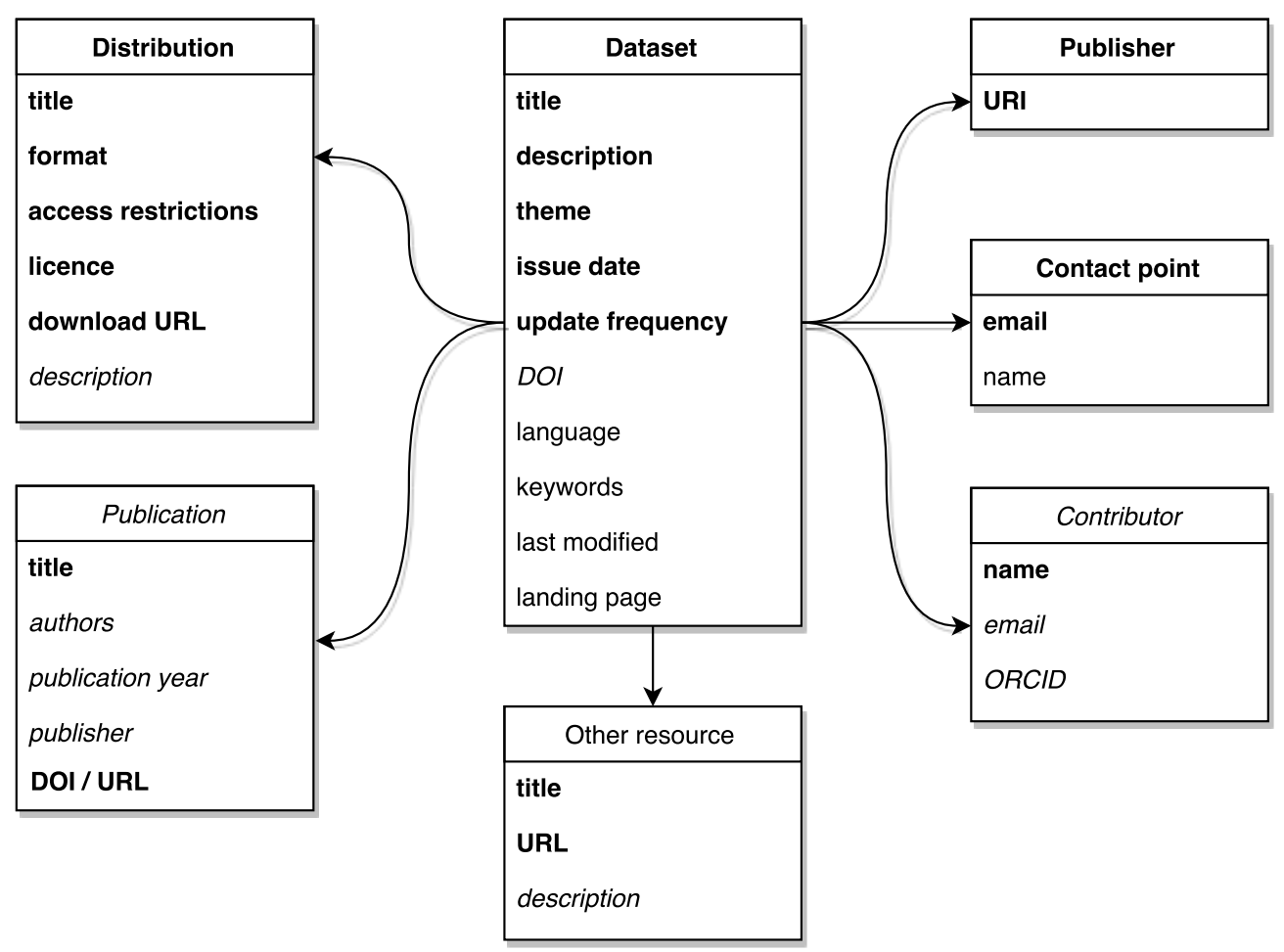

Figure 1: The JRC metadata schema at a glance. The fontfaces used denote whether the relevant resource or attribute is mandatory (bold), recommended (italic), or optional (normal).

may denote a data dictionary, input data, models, as well as legal acts and impact assessments.

Whenever possible, persistent URIs are used in order to ensure effective linking of the resources described in metadata records. These include identifiers used in the scientific community, such as DOIs for publications and datasets, and ORCIDs for researchers, but also persistent URIs such as the European Legislation Identifier (ELI) [19]. These identifiers are exploited also during the presentation of the dataset descriptions.

Moreover, each dataset in the JRC Data Catalogue is assigned a persistent URI from the URI registry of EU institutions and bodies operated by the Publications Office of the European Union (http: //data.europa.eu/). This will ensure the long-term availability of metadata records also in case the existing infrastructure is migrated.

It is important to note that, besides the linking requirement, persistent URIs are crucial for ensuring consistency in the metadata management workflow, since, based on them, it is possible to unambiguously detect changes of status of metadata records (new, deleted, updated records).

\section{A HIGH-LEVEL ARCHITECTURE FOR A MULTI-DISCIPLINARY DATA INFRASTRUCTURE}

This section presents the JRC Data Infrastructure, which implements the JRC Data Policy and ensures access to JRC datasets.

The architectural design was driven by requirements that include:
Single access point at the JRC level Access to the JRC data should be sustained and provided through a single access point at the JRC level [2, Art. 11(1)]. This access point is the JRC Data Catalogue, available at: http://data.jrc.ec.europa.eu.

Single JRC data channel to the EU Open Data Portal The JRC single access point shall be the only channel to make available the JRC data in the EU ODP (http://data.europa.eu/ euodp) set up in accordance with Article 5 of the Commission Decision on the reuse of Commission documents [2, Art. 11(2)].

EU ODP compatibility It shall be possible to automatically exchange metadata records with the EU ODP.

Data access and download. Data access endpoints and, when possible, direct download of datasets should be provided.

Metadata harvesting and analysis tools The access point should be able to automatically harvest metadata records from existing systems, thereby reducing the JRC data documentation efforts.

Open Source adoption According to the "Open Source Strategy" in the European Commission [9], open source software should be adopted.

General purpose data catalogue development The unique access point should offer the possibility to document any kind of datasets (e.g., geographical, statistical, biological, economic, etc.).

Figure 2 presents an overview of the JRC Data Infrastructure. The architecture is multi-tier and follows the Model-View-Adapter 


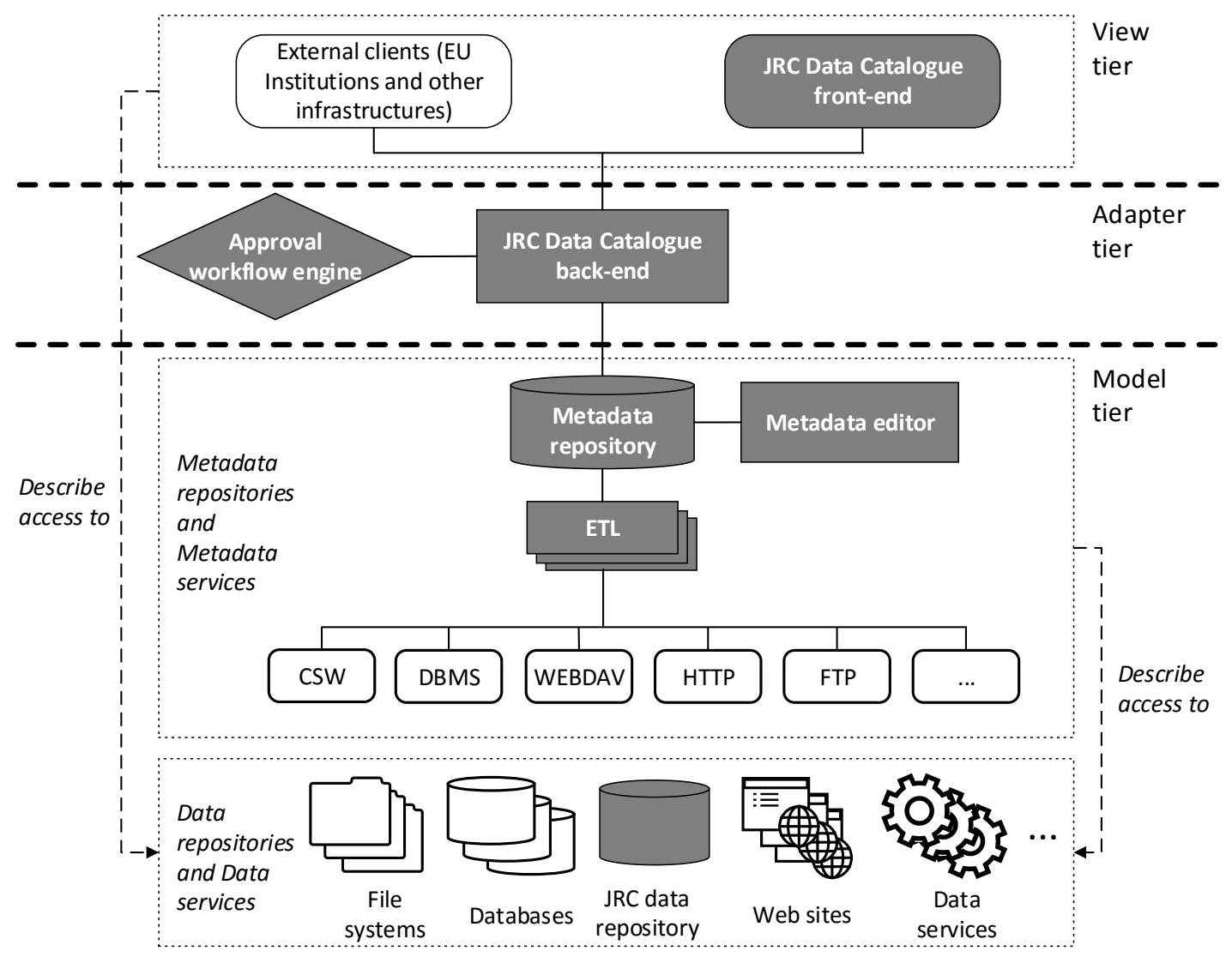

Figure 2: Architectural overview of the JRC Data Infrastructure. The grey components have been developed/adopted in-house in order to support the JRC Data Infrastructure.

(MVA) software architectural pattern (https://en.wikipedia.org/w/ index.php?title=Model-view-adapter). In particular:

View. This tier allows for the presentation of the information and the interaction with the end-users through different channels, including the JRC Data Catalogue front-end, and other external infrastructures (such as the EU ODP and other thematic community portals). The JRC Data Catalogue front-end is based on the Web client provided by the open source CKAN platform (http://ckan.org/). It offers various tools that currently include search functionalities (i.e., by keyword, by tag cloud, by collection of datasets), as well as detailed Web pages, i.e., dataset metadata, collections of datasets, FAQ and contact details. It also exposes an API for accessing the metadata.

Adapter. This tier interacts with both the View and Model tiers, and it is implemented by the JRC Data Catalogue back-end and the institutional approval workflow engine.

Approval workflow engine. This component supports the internal approval procedure for any JRC output, datasets included.

IRC Data Catalogue back-end. The main idea behind the JRC Data Catalogue back-end is to act as a flexible middleware acting between the metadata repository, the approval workflow engine, and the JRC Data Catalogue front-end as well as the EU ODP. It consists of different components including the CKAN back-end, which provides, among other features, an indexing and search component based on Apache Solr.

Model. This tier includes the data and metadata that are addressed by the JRC Data Infrastructure as well as the components that are managing them. The model tier allows distributed data and metadata management by the different groups within the JRC; these groups usually have their own data repositories and have two options for populating the metadata repository: (a) directly using a metadata editor; or (b) deploying an ETL ("Extract, Transform and Load") process, that can extract metadata from existing systems. For the few groups that have not established a data repository, the JRC Data Infrastructure provides a common data repository. The following components are part of this tier:

Data repository. This repository provides a simple and basic environment to upload datasets and related metadata. It gives public access to resources via both FTP and HTTP.

Metadata repository. This component is in charge of storing and maintaining the metadata records that describe the JRC datasets. The metadata records are either generated by the ETL or manually created using a metadata editor (both described below). The Metadata repository provides an environment where metadata produced 
by the users can be syntactically and semantically validated and previewed before publishing them on the JRC Data Catalogue. The storage of metadata is performed by the back-end that provides a REST API. A Web application built on top of the REST API of the back-end manages the metadata.

ETL. Through the ETL, the metadata repository can automatically harvest metadata records from various JRC metadata resources. The implementation of the ETL relies on the open source software Pentaho Kettle, within which ad hoc ETL modules were developed to support different endpoints (such as catalogue services, DBMSs, WebDAV folders, HTTP Web sites, and FTP servers) and transform different metadata source schemas to the JRC metadata schema.

Metadata editor. In order to allow users that do not have proper tools to document their datasets, a metadata editor was developed, that allows the users to create and maintain dataset metadata records to be published on the metadata repository. The generated metadata records are based on the JRC metadata schema.

\section{CONCLUSIONS}

In this paper we have presented various initiatives in place at the JRC in order to facilitate open access to research data according to the JRC Data Policy. In order to successfully reach these goals, we believe that a hybrid approach, having both top-down and bottomup components, is needed.

In order to address the heterogeneous nature of the JRC with its multidisciplinary research activities, we highlight the specific tailored JRC metadata schema and JRC Data Infrastructure architecture. The JRC metadata schema has been developed with the main requirements to be in line with current European and international standards, and at the same time to support the needs of the researchers at the JRC. Moreover, data citation is introduced to create an important incentive for the researchers to provide and maintain metadata. We believe this is essential for the implementation of the JRC Data Policy.

A final important initiative is the establishment of a coherent data architecture that is non-intrusive on existing data and metadata production workflows. By employing Semantic Web and Linked Data technologies for metadata, the architecture supports the heterogeneous nature of existing solutions for the management and dissemination of data. A key component is the ETL, which ensures that already existing metadata are aligned with the proposed JRC metadata schema and represented on the JRC Data Catalogue. Perhaps more importantly, it also allows metadata to be harvested from various legacy systems, thereby ensuring that metadata are only created once and maintained close to their source. This practice facilitates the implementation of the data policy for existing systems, and, as such it can be useful for other multidisciplinary organisations aiming to implement a coherent data infrastructure.

In future work we will further integrate the JRC Data Catalogue with other services, in order to consistently support the (possibly automated) linking between data and resources, such as models, legal acts, and impact assessments, which would facilitate data reproducibility and would enable a better understanding of how data are used and in which contexts.

\section{ACKNOWLEDGMENTS}

The authors wish to thank all the colleagues from the JRC who have been involved in the development of the implementation guidelines and in the concrete implementation of the JRC Data Policy. Special thanks go to Łukasz Wojciech Ziemba, Alessandro Dalla Benetta, Roberto Sgnaolin, and Alberto Di Taranto, who have contributed to the implementation of components for the JRC Data Infrastructure.

\section{REFERENCES}

[1] DataCite Metadata Working Group. 2016. DataCite Metadata Schema for the Publication and Citation of Research Data. Version 4.0. DataCite Specification. DataCite. https://doi.org/10.5438/0012

[2] Catherine Doldirina, Anders Friis-Christensen, Nicole Ostländer, Andrea Perego, Alessandro Annoni, Ioannis Kanellopoulos, Massimo Craglia, Lorenzino Vaccari, Giacinto Tartaglia, Fabrizio Bonato, Jean-Paul Triaille, and Stefano Gentile. 2015. FRC Data Policy. EUR - Scientific and Technical Research Reports. European Commission, Joint Research Centre (JRC). https://doi.org/10.2788/607378

[3] European Commission. 2008. Commission Regulation (EC) No 1205/2008 of 3 December 2008 implementing Directive 2007/2/EC of the European Parliament and of the Council as regards metadata (Text with EEA relevance). Official fournal of the European Union 51, L 326 (Dec. 2008), 12-30. http://data.europa.eu/ $\mathrm{eli} / \mathrm{reg} / 2008 / 1205 / \mathrm{oj}$

[4] European Commission. 2011. 2011/833/EU: Commission Decision of 12 December 2011 on the reuse of Commission documents. Official fournal of the European Union 54, L 330 (Dec. 2011), 39-42. http://data.europa.eu/eli/dec/2011/833/oj

[5] European Commission. 2012. 2012/417/EU: Commission Recommendation of 17 July 2012 on access to and preservation of scientific information. Official fournal of the European Union 54, L 194 (July 2012), 39-43. http://data.europa.eu/eli/reco/ 2012/417/oj

[6] European Commission. 2012. Towards better access to scientific information: Boosting the benefits of public investments in research. $\operatorname{COM(2012)~} 401$ final. 1-12 pages. http://eur-lex.europa.eu/legal-content/EN/ALL/?uri=COM:2012:0401:FIN

[7] European Commission. 2013. EU implementation of the G8 Open Data Charter. (Oct. 2013). https://ec.europa.eu/digital-single-market/en/news/ eu-implementation-g8-open-data-charter

[8] European Commission. 2015. Better Regulation Toolbox. (2015). https://ec. europa.eu/info/files/better-regulation-toolbox_en

[9] European Commission. 2015. Open Source Strategy in the European Commission. (March 2015). http://ec.europa.eu/dgs/informatics/oss_tech

[10] European Union. 2013. Regulation (EU) No 1291/2013 of the European Parliament and of the Council of 11 December 2013 establishing Horizon 2020 - the Framework Programme for Research and Innovation (2014-2020) and repealing Decision No 1982/2006/EC (Text with EEA relevance). Official fournal of the European Union 56, L 347 (Dec. 2013), 104-173. http://data.europa.eu/eli/reg/2013/1291/oj

[11] ISA DCAT-AP Working Group. 2015. DCAT Application Profile for European Data Portals. Version 1.1. ISA Specification. EU ISA Programme. https://joinup.ec. europa.eu/asset/dcat_application_profile/asset_release/dcat-ap-v11

[12] ISA GeoDCAT-AP Working Group. 2015. GeoDCAT-AP: A geospatial extension for the DCAT application profile for data portals in Europe. Version 1.0. ISA Specification. EU ISA Programme. https://joinup.ec.europa.eu/asset/dcat_application_ profile/asset_release/geodcat-ap-v10

[13] ISO/TC 154. 2013. ISO 17369:2013 Statistical data and metadata exchange (SDMX). ISO Standard. International Organization for Standardization (ISO). https://www. iso.org/standard/52500.html

[14] ISO/TC 211. 2003. ISO 19115:2003 Geographic information - Metadata. ISO Standard. International Organization for Standardization (ISO). https://www.iso. org/standard/26020.html

[15] Fadi Maali and John Erickson. 2014. Data Catalog Vocabulary (DCAT). W3C Recommendation. W3C. https://www.w3.org/TR/vocab-dcat/

[16] Andrea Perego, Anders Friis-Christensen, and Michael Lutz. 2016. GeoDCATAP: Use Cases and Open Issues. In Workshop on Smart Descriptions \& Smarter Vocabularies (SDSVoc). https://www.w3.org/2016/11/sdsvoc/SDSVoc16_paper_25

[17] Andrea Perego, Anders Friis-Christensen, Lorenzino Vaccari, and Chrisa Tsinaraki. 2016. Using DCAT-AP for Research Data. In Workshop on Smart Descriptions \& Smarter Vocabularies (SDSVoc). https://www.w3.org/2016/11/sdsvoc/ SDSVoc16_paper_27

[18] Andrea Perego, Anders Friis-Christensen, Lorenzino Vaccari, and Chrisa Tsinaraki. 2017. DataCite to DCAT-AP Mapping. Technical report. European Commission, Joint Research Centre (JRC). https://webgate.ec.europa.eu/CITnet/stash/ projects/ODCKAN/repos/datacite-to-dcat-ap/

[19] Publications Office of the European Union. 2016. European Legislation Identifier (ELI). (2016). http://publications.europa.eu/mdr/eli/ 